\title{
Concept of Socio-Cyber-Physical Work Systems for Industry 4.0
}

\author{
Elvis HOZDIĆ, Peter BUTALA
}

\begin{abstract}
In this paper the concepts of advanced production systems based on the challenges that bring the new industrial revolution named - Industry 4.0 are presented. The presented concept of socio-cyber-physical work systems is based on connecting social, cyber and physical working environments into a single functional, productive entity of the appointed elementary socio-cyber-physical work system. The elementary socio-cyber-physical work system is a basic building block of the cyber-physical production systems at the manufacturing level. The cyber system of the elementary socio-cyber-physical work system enables autonomous decision-making and cooperation in the network system. The possibility of implementing the proposed concept is based on the introduction of agency technologies in the domain of modern production systems and the development of information-communication technologies for the advanced management and control of cyber-physical production systems. Some illustrative examples reflect the experimental results of a research work in the field of cyber-physical systems and demonstrate the potential possibilities of implementing the concept of sociocyber-physical work systems in the real industrial environment.
\end{abstract}

Keywords: agent; cyber-physical system; Industry 4.0; internet of service; internet of things

\section{INTRODUCTION}

In the era of globalization, scientific discoveries and technological advancements, which significantly influence today's manufacturing industries, it is time to rethink the way of how industrial products are manufactured. A novel approach to industrial production is needed, which would address new challenges and circumstances arising from escalating uncertainty, dynamism and complexity in industry driven by turbulent market, political, economic and societal conditions.

A new production philosophy, which has emergesd recently under the name of Industry 4.0, opens space for novel approaches to industrial production [1-7].

Industry 4.0 is driven by new discoveries of sciences, enriched knowledge, new and enhanced materials and technologies, especially in the field of information and communication technologies (ICT). Besides, novel organizational forms and innovative managerial principles of emergence, self-organization, learning, open innovation, collaboration, and networking of humans and organizations will become the key elements of the next generation manufacturing systems.

Recently, a new approach is emerging in terms of a cyber-physical production system (CPPS) [8], which upgrades in a way the mentioned attempts. It originates from the concept of cyber-physical systems (CPS) [9], and links physical and virtual components of a production system into a coherent whole. The concept of CPPS [10] merges the functionalities and benefits of CPS applied to the industrial context.

The aim of this paper is the conceptualization of a socio-cyber-physical work system (SCPWS) for Industry 4.0.

This work arises from the previous research in agentbased systems [11] and distributed manufacturing systems [11], and is based on the concept of cyber-physical production systems (CPPS) [12]. The paper presents the evolutionary development of the concept of adaptive distributed production systems (ADMS) [13]. Within CPPS [12] the elementary work systems (EWS) [14] are defined as building blocks. In this paper, the EWS is restructured in the spirit of the CPS.
The main question here is how the elements of the new structured EWS and their relatioships are defined and how the new structured EWS participates cooperatively in network systems. The proposed concept shows developed communication interfaces and communication between the production process participants in the cyber system of CPPS to the management and control of manufacturing systems in the real time.

The rest of the paper is organized as follows. In Section 2 a brief literature review of enabling concepts and technologies is presented. Section 3 introduces the models of the elementary socio-cyber-physical work system (ESCPWS). Section 4 describes the cyber system infrastructure for implementation of ESCPWS. In Section 5 , the potential implementation of the ESCPWS in the industrial environment is presented. Section 6 summarizes the work carried out and makes suggestions for future research.

\section{LITERATURE REVIEW}

In the next, a brief overview of literature related to enabling technologies and underlying concepts for CPPS is presented.

\subsection{Enabling Technologies}

Manufacturing has always been on the front end of technological advancements while generating wealth. And there has always been an open question of how to generate it faster and cheaper. Therefore, the potential of new information and communication concepts and technologies, such as internet of things, internet of services, cloud computing, smart environments, social media, agent technology. Last but not least, cyber-physical systems have been recognized and there are several ongoing research projects, which try to facilitate the implementation and integration of new technologies with the objective to develop more adaptable and responsive production systems. 


\subsubsection{Internet of Things}

The Internet of Things (IoT) is a computing concept that describes a future where every day physical objects will be connected to the Internet and will be able to identify themselves to other devices. The term is closely identified with Radio Frequency Identification (RFID) as the method of communication. However, it also may include other sensor technologies, wireless technologies or Quick Response (QR) codes.

The IoT is a combination of technological push and a human pull for more and ever-increasing connectivity with anything in the immediate and wider environment. This push-pull combination makes the development very strong, unstoppable, fast and extremely disruptive. It is foreseen that within the next decade the internet will exist as a seamless fabric of classic computer networks and networked objects. Content and services will be all around us, always available, paving the way to new applications, enabling new ways of working, interacting, entertaining and living. This innovation is enabled through embedding of digital electronics into everyday physical objects, making them "smart" and letting them seamlessly integrate within the global resulting cyber-physical infrastructure [15].

The smart objects will have technological capabilities which will allow communication, identification and processing interaction and will become active participants in business, information and social processes. They will communicate with each other, exchange information with the environment and receive information from sensors and independently react to events and will be influenced by running processes that initiate actions and provide services with or without direct human intervention [16].

\subsubsection{Internet of Service}

The basic idea of the Internet of Services $(I o S)$ is to systematically use the Internet for new ways of value creation in the services sector. There are different angles from which one may look at this approach. From an IT perspective, cloud computing, service oriented architectures (SOA), software-as-a service (SaaS), platform-as-a-service (PaaS) and infrastructure-as-aservice (IaaS), as well as business process outsourcing are related trends. SaaS, PaaS and IaaS [17] are three common types of service delivery models. These services are usually delivered from computational clouds through standard interfaces, such as web services and SOA.

The manufacturing industry is undergoing a major transformation enabled by information technology and related smart technologies. Cloud computing is one of such smart technologies [17]. The National Institute of Standards and Technology (NIST) [18] defined cloud computing as: "a model for enabling ubiquitous, convenient, on-demand network access to a shared pool of configurable computing resources (e.g., networks, servers, storage, applications, and services) that can be rapidly provisioned and released with minimal management effort or service provider interaction".

Mirroring NIST's definition of cloud computing, cloud manufacturing may be defined as "a model for enabling ubiquitous, convenient, on-demand network access to a shared pool of configurable manufacturing resources (e.g., manufacturing software tools, manufacturing equipment, and manufacturing capabilities) that can be rapidly provisioned and released with minimal management effort or service provider interaction" [17].

\subsubsection{Cyberspace}

Over the years, many different definitions have evolved for cyberspace. Cyberspace is defined as: "an infinite artificial world where humans navigate in information-based space" and as "the ultimate computerhuman interface" [19].

The US Department of Defence considers cyberspace as: "a global domain within the information environment consisting of the interdependent network of information technology infrastructures, including the internet, telecommunications networks, computer systems, and embedded processors and controllers" [20]. This definition only refers to the (hardware) technology components, although software and data may be inferred from the wording.

According to [21] cyberspace is a time - dependent set of interconnected information systems and the human users that interact with these systems. A distinctive and constitutive feature of cyberspace is that no central entity exercises have control over all the networks that make up this new domain. Cyberspace is a virtual world in which circulating digital data is being exchanged in the communication between participants.

\subsubsection{Multi-Agent Technology}

The agent-based technology is a promising technology for self-organizing control of production systems. An agent is defined as a software system that communicates and cooperates with other software systems to solve a complex problem that is beyond the capability of each individual software system [22]. According to [23] an agent has four basic functional elements: perceptor, evaluator, effector, and inference mechanism. The role of the perceptor is: (1) to observe a state of the environment in order to recognize information relevant to the agent, (2) to form inputs from this information, and (3) to trigger the evaluation process. The inference mechanism controls the evaluation by reasoning. The reasoning process is based on embedded data and knowledge with the reference to a set of goals. Output is launched into the environment by the effector and thus affects the state of the environment.

In the domain of agent production control system, Leitão [24] defines an agent in the following way: an agent is an autonomous component that represents physical or logical objects in the system, capable to act in order to achieve its goals, and being able to interact with other agents when it does not possess knowledge and skills to reach alone its objectives.

Fundamental characteristics of agents, such as reactivity, proactivity, autonomy, cooperation, adaptability and mobility, are defined in the works [22].

An agent may operate in an environment that includes other agents. The community of interacting agents, as a whole, operates as a multi-agent system (MAS). MAS are formed as a network of computational agents that interact 
and typically communicate with each other. MAS can be considered as a system that is asynchronous, stochastic and distributed.

Local autonomy and self-organization are the key attributes of agent in the CPPS. The current state of the industrial applications of agent technology in CPPSs clearly indicates that software agents can effectively address arising CPPS challenges [25, 26].

\subsection{Cyber-Physical Systems}

Cyber-physical systems (CPS) are a new generation of systems that integrate computer and physical capabilities [27].

Using the appropriate sensor and actuator technologies, these systems are able to receive direct physical signals and convert them into digital data and, on the basis of such information, enable management of systems in real time. They can share collected data in the form of information and make them available through digital networks, making in that way the Internet of Things [28].

CPSs will provide multiple possibilities in different application domains where time plays a crucial role, such as the electricity distribution grids, medical devices and systems, the industrial control and monitoring systems, the environmental control, precision farming, the advanced automotive systems, traffic control, the aircraft control systems, distributed robotic systems, defense systems, as well as the production systems.

On this basis, cyber-physical production systems (CPPS) are defined as systems that use CPS-related technology, including various electronic devices embedded in different network of processing and communicating elements, with the aim to increase production system flexibility, adaptability and efficiency in complex production environment [29, 30].

CPPS will also be capable of analyzing data in realtime and of making decisions autonomously or via humanmachine interaction thus taking advantage of specific capabilities of humans and CPSs [31].

In a CPPS, the horizontal integration is accomplished through value networks and the vertical integration is achieved through networked manufacturing systems [32, 33]. The integration can be realized by the use of different technologies, such as Multi-Agent Systems (MAS), Service-Oriented Architecture (SOA), Internet of Things (IoT), cloud computing, augmented reality, Big Data, Machine-toMachine (M2M) communication, and similar [32].

The emergence of CPSs and cross-linked CPPSs will lead to fundamental restructuring of manufacturing work and logistic systems and will require new forms of humanmachine interaction [34].

CPPSs partly break with the traditional automation pyramid. The typical control and field levels, which include common PLCs (programmable logic controllers) close to the technical processes providing the highest performance for critical control loops still exist, while on the other, higher levels of the hierarchy a more decentralized way of functioning is characteristic in CPPS [8].
Production processes in cyber spirit were modeled by Peklenik [14]. Peklenik defined an elementary work system (EWS) as a basic building block of manufacturing systems. EWS consists of a process, process implementation device (PID) and a (human) Subject. A Subject here may be an individual or a team. Thus, EWS represents a socio-physical system. In the case that PID is computer controlled, the controller may be connected to a local area network, which represents a gateway to the cyberspace. With the concept of the EWS the basic level of the production system is defined and it enables the structural complexity of the manufacturing system.

By definition [14], EWS has the task of transformation of inputs into outputs in order to achieve more value directly at the process level. This function is not to indirectly create a new value based on the feed. In order to function the EWS enriches the advanced functions of feeding controls, Butala and Sluga [11] define virtual work system (VWS).

As its counterpart for representing EWS in the cyberspace, a virtual work system (VWS) was introduced [11]. VWS acts as the EWS's agent on the internet, i.e. in the cyberspace. EWS and VWS are connected as depicted in [11]. Thus, the tandem of EWS and VWS form together a cyber-physical work system, which can be considered as an origin for developing building blocks of the future CPPS.

In what comes next, a concept of a social-cyberphysical work system (SCPWS) is presented.

\section{CONCEPT OF SOCIO-CYBER-PHYSICAL WORK SYSTEM (SCPWS)}

The model of the basic building block of the sociocyber-physical work system (SCPWS) is presented in Fig. 1.

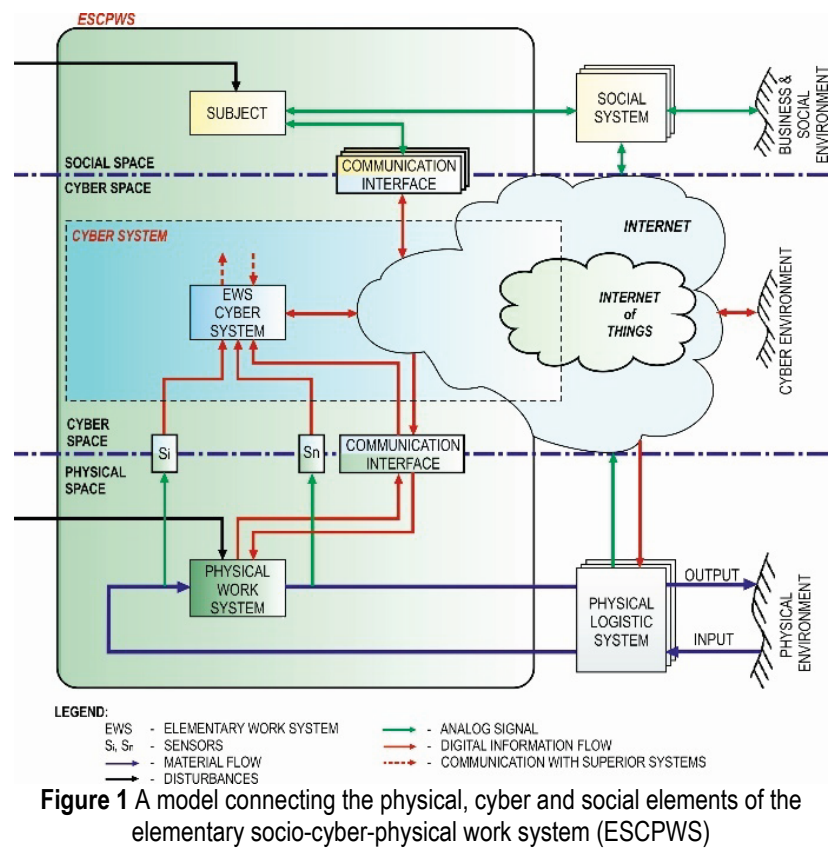

The basic building block of the SCPWS is elementary socio-cyber-physical work system (ESCPWS) (Fig. 1).

The model of ESCPWS is structured based on the generic model of CPPS [12] with the three constitutive 
elements: the Subject, the physical work system (PWS), and cyber system of the elementary socio-cyber-physical work system (EWS cyber system).

The PWS structure of the ESCPWS is based on elementary work system (EWS) structure $[12,14]$.

The EWS cyber system is defined in [35]. The EWS cyber system enables development and implementation of new functions in ESCPWS (monitoring, self-organization, self-adaption, self-diagnostic, etc.), (4) vertical connection of ESCPWS in integrated work structure (AWS, PBS, service network, production network), and (5) horizontal connection of ESCWS in the Internet of Things network. The EWS cyber system structure is shown in Fig. 2.

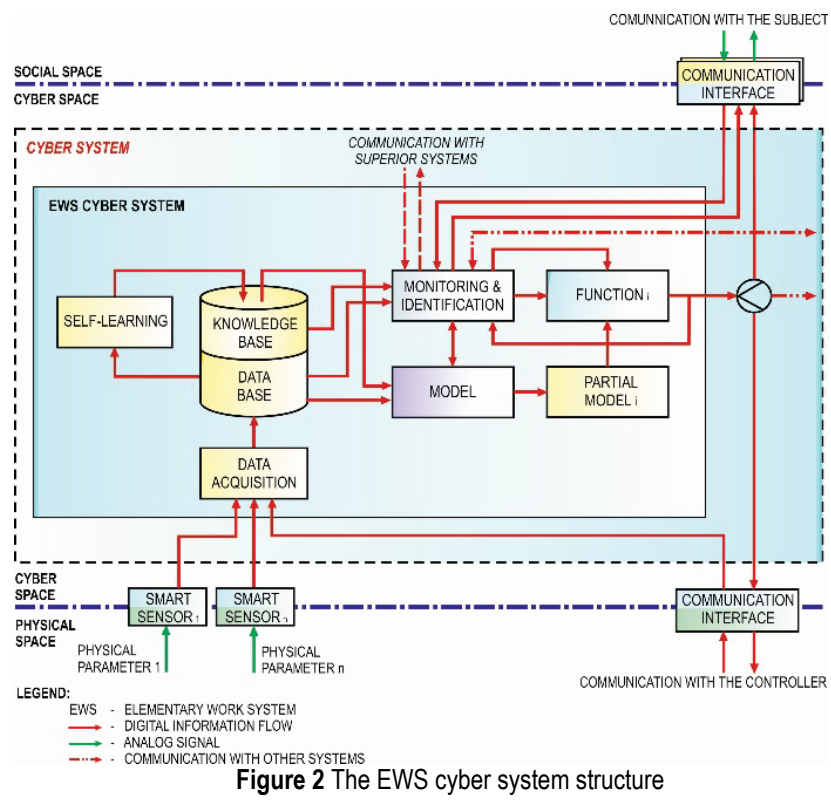

The monitoring and identification in the EWS cyber system enables: 1) monitoring and display system operating status of individual elements of a physical system, 2) triggering alarms in case of exceeding the set tolerance limits with the individually monitored size, etc.

Support for implementation of monitoring and identification in the cyber system of ESCPWS is represented by models stored in the knowledge base. Model knowledge provides information about the structure of the system, the elements of a PWS, on the current state of the elements of a PWS based on current data and the like.

In the EWS cyber system, it is possible to implement "smart" functions specific to a particular ESCPWS. The "smart" functions such as self-diagnostics, selforganization, self-adaptation, self-maintenance, selfcalibration, self-correction, etc., represent functions for managing and controlling ESCPWS in the real-time. The input values of individual functions represent data from the physical environment stored in the knowledge and database, information from superior systems (AWS or EWS networks), etc.

The function self-adaptation is based on the concept of adaptive process control described in the paper [36]. The adaptive function of process parameters has a role of adaptation of reference values parameters of process $(R)$. Input values in the function of adaptation represent data obtained from involving physical environment. Adaptive function of process parameters is supported by target values and rules that are generated through the performance of a loop of self-learning. Exit functions are new, adapted reference values of process parameters $\left(R_{\mathrm{A}}\right)$. $\left(R_{\mathrm{A}}\right)$ is forwarded to the $\mathrm{LC}$ as a new reference for a future cycle process.

The proposed concept of adaptive control process is based on connecting information about the current state of the manufacturing process, information from previous production process and the information obtained from the prediction of the future state of the manufacturing process (Fig. 3). The adaptation function is presented in Section 5.

The Subject's actions are minimized on the operation level, and the management and control of ESCPWS is implemented by advanced ICT (eg, certain rules, algorithms, etc.). The communication of Subject with the EWS cyber system is enabled through the communication interfaces. An example of a communication interface is shown in Section 4.

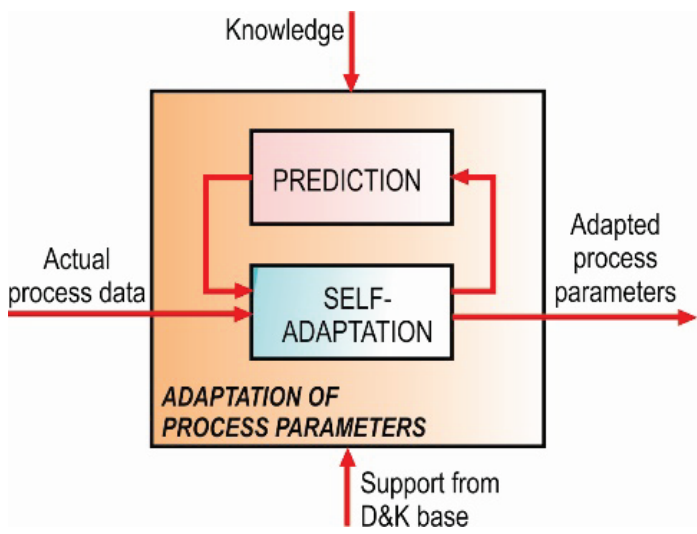

Figure 3 The function of adaptive process control in the ESCPWS

Outputs from the EWS cyber system represent the outputs of individual "smart" functions, which are implemented in the EWS cyber system. These functions can be run independently of each other, they can run at the same time, in parallel, and aim to support the management and control of the ESCPWS.

The vertical connectivity of the EWS cyber system includes ESCPWS in superior structures such as AWS cyber system or PBS cyber system. The horizontal connectivity of the EWS cyber system with other systems in operative level includes ESCPWS in the Internet of Things network. In this way, the communication between a different ESCPWS is based on a multi-agent communication.

In the next section, the infrastructure for implementation of the developed concept of the ESCPWS is presented.

\section{THE INFRASTRUCTURE FOR IMPLEMENTATION OF SCPWS}

The control system defines the production turn and determines when and in what quantities the individual products are made.

For the implementation of the distributed approach to control and management of manufacturing systems Monostori et al. in the article [37] suggest the idea to add an agent, or a program entity, to an element of the physical 
or electronic world, which will represent such an element in the digital, cyber world. This enables the agent to participate in the distributed management and control of manufacturing and working systems.

MASs are seen as a key enabler in the development of CPPSs, as they support the design of distributed control systems based on the allocation of control functions to autonomous and cooperative agents [24].

In the EWS cyber system, software agents and multiagent systems are the actors involved in interactions. The agent-based infrastructure of the cyber system is presented in Fig. 4.

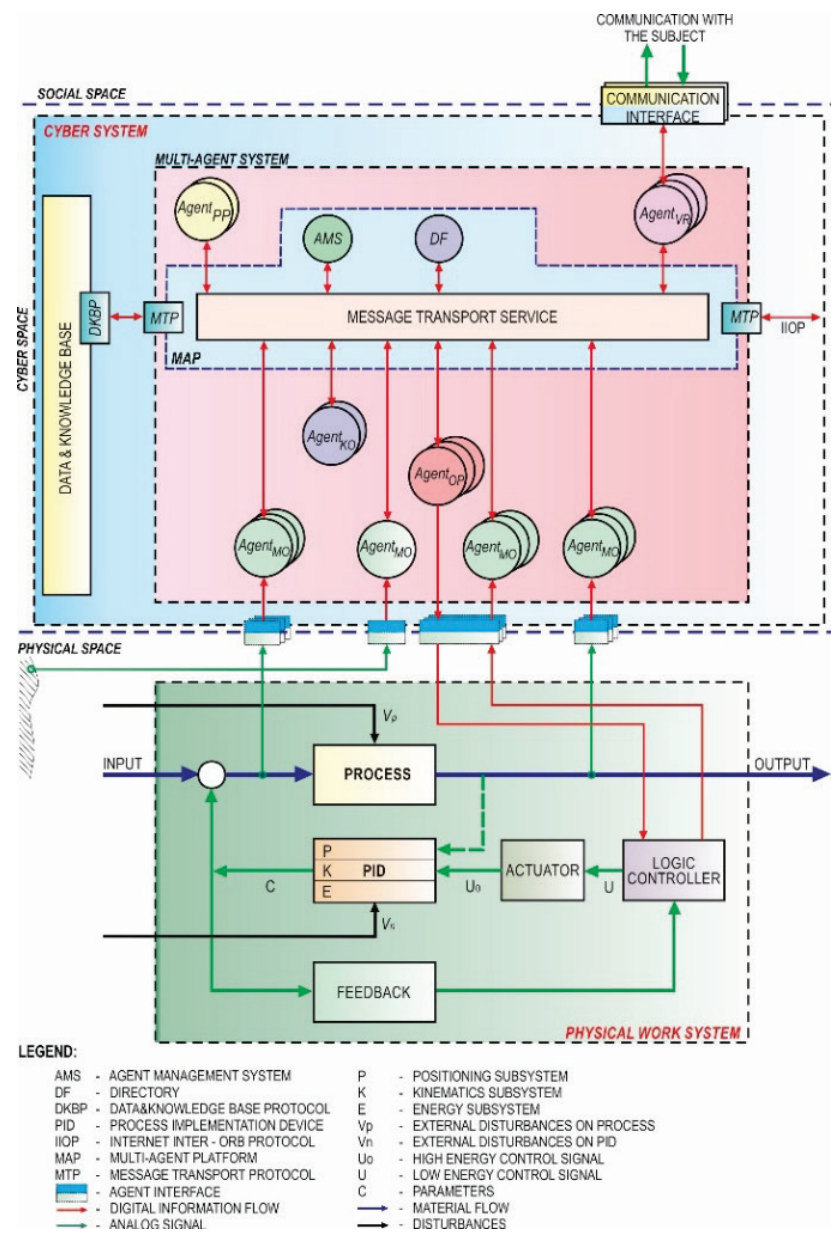

Figure 4 Architecture of multi-agent based model for distributed management and control of SCPWS

In Fig. 4, the physical elements are present in the form of PWS. The management and control of these physical elements is carried out via a real-time controller or the logic controller (LC). The LC represents an element of the physical system, which is based on the incoming input signals received from the input devices, according to a specific program, from the output signals controlled by the output devices. The LC is programmed with a dedicated programming language, based on IEC 61131-3 standard and which was extended by it for the needs of distributed management and control (IEC 61499) [38]. Connecting LCs with a multi-agent system (Fig. 4) is enabled through the agent interface, which represents the interface between physical and cyber systems.

Connecting a multi-agent system with a standard communication functional block of bidirectional transmission of CLIENT / SERVER, that is based on communication protocol -User Datagram Protocol (UDP) that enables bidirectional communication between operational and executive work system functions [39]. This means that the production parameters will be used in the adaptive decision-making process in real-time, which ensures the machining within optimal tolerance limits.

The infrastructure of the cyber system compiles D\&K bases and agent structures linked to a multi-agent system. In the multi-agent system, agent structures are presented: 1) agents of the PBS cyber system $\left(\right.$ Agent $\left.\left._{P B S}\right), 2\right)$ agents of the coordination\& collaboration level of cyber system $\left(\right.$ Agent $\left.\left._{K O}\right), 3\right)$ agents of the operative level in the cyber system $\left(\right.$ Agent $\left.\left._{O P}\right), 4\right)$ agents that realize the monitoring activity in the cyber system $\left(\right.$ Agent $\left._{M O}\right)$, and 5) agents that enable the communication Subject - cyber system $\left(\right.$ Agent $\left._{V R}\right)$.

Agent $_{P B S}$ are the agents that enable the management and control of CPPS on the business level of cyber system. They represent digital mechanisms for implementing "smart" functions in PBS cyber system of CPPS. A typical representative of such agents is the agent in charge of carrying out the production planning function $\left(\right.$ Agent $\left._{P r P}\right)$.

The agents of the coordination \& collaboration level of cyber system are digital mechanisms for the implementation of management and control functions in the AWS cyber system, such as the transport function (transport agents - Agent $_{T R}$ ), the maintenance function of autonomous work systems (maintenance agent - ggent $_{M}$ ), the technology design function (design agent - Agent $_{\text {Tech }}$ ), quality control function (quality agents - $A g e n t_{C C}$ ), job scheduling function (scheduling agent - Agent $_{S c h}$ ), etc.

Agent $_{O P}$ are agents that exist in the EWS cyber system and have the role of the digital mechanisms for implementing functions within the EWS, such as the selforganization function (self-organizing agent - gent $_{S O}$ ), self-calibration function (self-calibration agent - Agent $_{S C}$ ), self-diagnostic function (self-diagnosis agent - $A g e n t_{S D}$ ), self-adaptation function (self-adaptation agent - Agent $_{S A}$ ), etc.

The agents that realize the monitoring activity in the cyber system $\left(\right.$ Agent $\left._{M O}\right)$ enable the monitoring of the state of the physical environment and its physical work systems. Based on the work [40], agent structures of monitoring in the cyber systems are: input agent (IA), output agent (OA), environment agent (EA), agent representing device in the cyber system - device agent (DA), and mediator agent (MA).

Agent $_{V R}$ enables presentation of the ESCPWS status to the Subject through the graphycal user interfaces formation (GUI) for visual activity monitoring of all agents in the MAS, as well as virtual presentation of information from the cyber system.

Agents need a specific Agent Framework for their creation and work. In addition to the environment agent, managesd by the life cycle agent, agents also need certain services that allow access to the required resources, as well as for the realization of complex algorithms. The environment agent enables messaging between agents, agents' mobility, and their protection against unwanted effects (unauthorized access to agents and services, protection against viruses, and the like). This means that agents are not communicating directly, but messages are 
transmitted through an environment agent that has mechanisms to select a particular agent for which the message is intended.

The message exchange between the agents, agents' mobility and their protection is ensured through the establishment of a program multi-agent platform and its information infrastructure in MAS. For the development of information infrastructure of cyber system in the SCPWS concept, a JADE environment and a JADE multi-agent platform with an infrastructure are prescribed by the FIPA standard. The JADE environment is written in the Java language program and uses the mobility system - the Remote Objective Invocation (RMI) and technologies for the implementation of distributed agent concepts Common Object Request Broker Architecture (CORBA).

The COBRA standard defines a framework in which objects are shaped, implemented on the server side. CORBA technology is based on the Internet Inter-ORB Protocol (IIOP) protocol. The systems are compiled by: CORBA application servers, or CORBA components executed on servers (e.g. D\&K bases, agents, services, and the like); IIOP protocol for communication between servers and users, and CORBA clients, who are responsible for implementing CORBA components on servers.

The agents of multi-agent system via its Message Transport Protocol (MTP) connect with D\&K database, based on the Data\&Knowledge Base Protocol (DKBP).

FIPA defines the JADE platform as a whole composed of individual components:

- a component that manages agents - Agent Management System (AMS),

- a component that takes care of setting up and extracting agent information - Directory Facilitator $(D F)$,

- a component that enables communication between agents within the agent environment - Agent Communication Channel (ACC),

- a component that connects individual components and sub-systems and enables their interworking - Internal Platform Message Transport (IMTP),

- a component that enables the connection of MAPs to other platforms and elements in the cyber system Message Transport Protocol (MTP).

The generic structure of the aforementioned and described agents, which exists in the multi-agent system, is based on the principles issued by the FIPA standards [41] and the principles set by the authors in the work [23]. In Fig. 5, the program agent structure is displayed by FIPA standard.

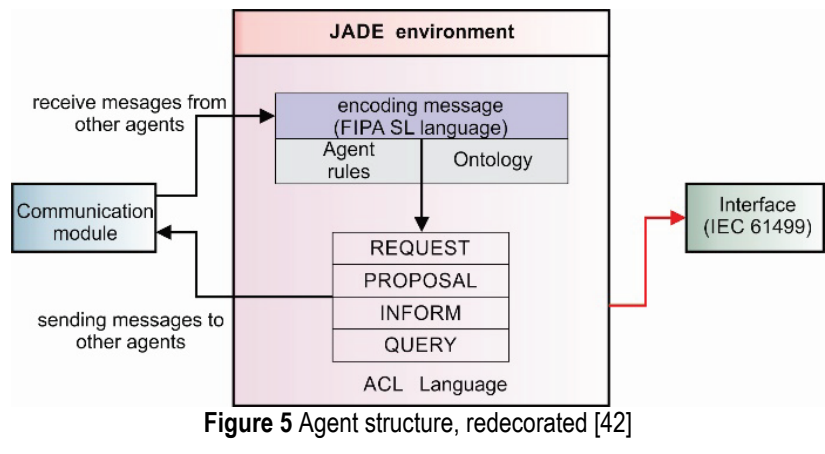

In what comes next, for the implementation of the cyber cystem, shown in Fig. 4, JADE program library [42] is used, that enables object-oriented programming in the Java programming language.

\subsection{Cyber System Implementation}

Implementing a JADE environment enables a distributed multi-agent platform. The platform is made of "containers", which contain agents. Containers represent an example of the JADE executive environment. Each container is basically a Java Virtual Machine (JVM), which provides a complete executive environment for agents and enables the competitive performance of agents that are implemented in the JADE environment as a special Java Threds. Such agent implementations allow parallel executing task agents in the multi-agent system.

For the implementation of the SCPWS concept, a multi-agent platform, including container with five containers, was developed: 1) the main container in which AMS, DF and reactive agents are located (agents that do not have symbolic environments in which they operate; they only react to excitement from environment), 2) the container-1 in which Agent PBS $_{\mathrm{BS}}$ are located, 3) the container-2 contains Agent $t_{\mathrm{VR}}$, 3) the container-3 containing Agent $_{\mathrm{OP}}$, 4) the container-4 where Agent $_{\mathrm{KO}}$ are located, and 5) the container-5 where Agent $_{\mathrm{MO}}$ are located (Fig. 6)

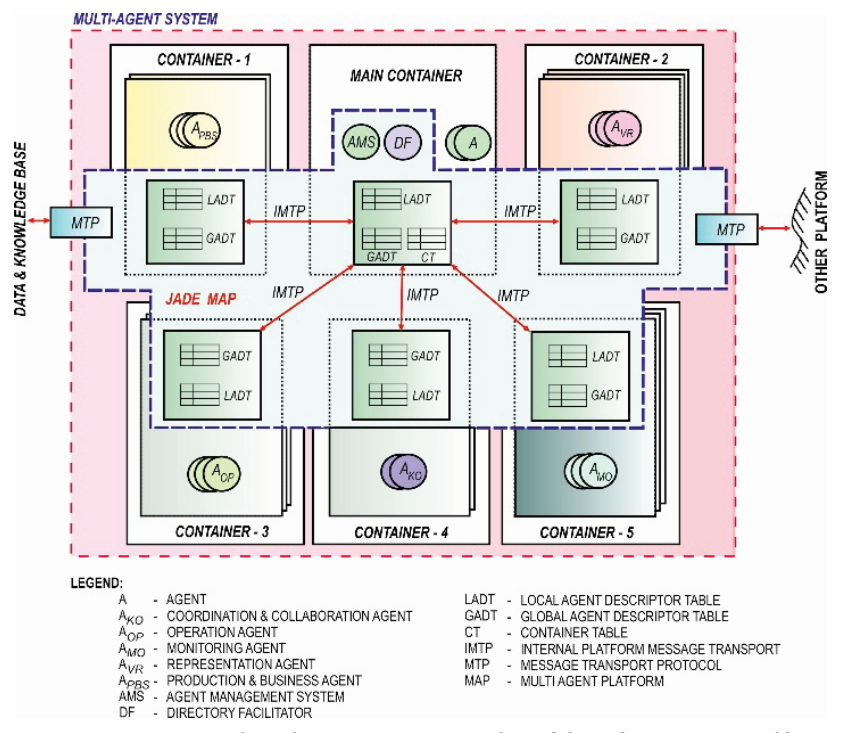

Figure 6 JADE platform for implementation of the SCPWS, adopted by [42]

In the main container, a Global Agent Descriptor Table (GADT) is placed in which all the representatives presented in the MAP are recorded including their current position as well as the Container Table (CT) position, which represents the register of reference objects and transport addresses of all other Accountants Assumed in the MAP. The JADE platform allows the establishment of local registers in the containers in the form of a Local Agent Descriptor Table (LADT).

The LADT register agent enables the agent of local expression to communicate with the other agent. In the example that this procedure fails, the agent addresses GADT in the main container. 
Communication between agents located in different containers is carried out through communication with the main container. For the communication of the main containers with other containers in MAP, the internal protocol for transport IMTP is in charge. IMTP is essentially different from MTP because it is not necessarily implemented entirely by FIPA standard, which enables improvement of the performance of the same MAP.

When establishing a JADE multi-agent platform, the main container is activated as the original MAP element, followed by all other established containers. The establishment of individual agents derives from the inheritance of the jade.core.Agent class, as shown in the example for the establishment of a device agent (DA):

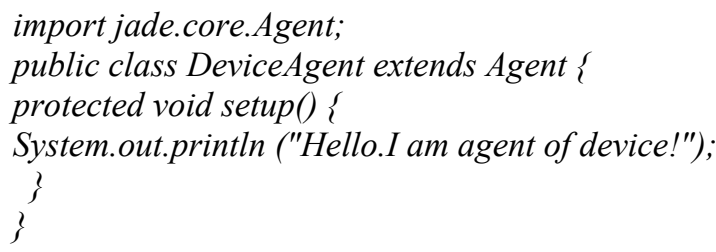

Jade.core.Agent class is a basic class for all agents and it provides all the elements necessary for an agency to establish interaction with MAP (registration, configuration, etc.) as well as appropriate methods that offer the implementation of typical agent activities (sending and receiving messages, using standard protocols, etc). When the agent is established its identification and initiation according to FIPA standard for JADE MAP agent starts.

In order for each agent to be simultaneously engaged in performing a number of different tasks, it is essential to enable a competitive performance of activities within the agent itself. The JADE multi-agent platform allows the use of classes that have impact on jade.core.behaviours. To make the agency implement its task, defined by the object Behavior, the agent should assign the behavior with method addBehavior (). The class defines two key methods that are used during task implementation for each agent: action(), which is used for task implementation, and done () that is in use to determine if the task implementation is complete.

The agent environment JADE contains in the advanced definitions class of heir, which define a wide range of tasks that can be accomplished by this mechanism:

- The ReceiverBehavior class is used to receive the message. It based on the method action () copies the message to the attribute, so it can be obtained by invoking the getMessage () method,

- The SimpleBehavior class is in charge of shaping simple tasks that cannot be interrupted. Its subclasses are:

- OneShotBehavior - defines a task that is performed only once,

- CycleBehaviour - defines a task that is performed cyclically,

- TickerBehaviour - defines a task that is performed periodically,
- WakerBehaiour - defines task implementation over a specific time period

- $\quad$ The CompositeBehaviour class defines complex task implementation. Its subclasses are:

- ParallelBehaviour - performing parallel tasks,

- SequentialBehaviour - performing tasks that follow each other.

Communication between agents in MAS based on JADE multi-agent platform is defined through asynchronous messaging. The basis for message exchange is the transmission of the $\boldsymbol{A C L M e s s a g e}$ class object. The structure of the message that the agents exchange is coordinated with Agent Communication Language (ACL) defined by FIPA. Messages sent from agent to agent have the following forms: INFORM - transmission of information, $\boldsymbol{R E Q U E S T}$ - request transmission for performing certain agent activities in the system, $\boldsymbol{Q U E \boldsymbol { E } R \boldsymbol { Y }}$ - questions transmission, and PROPOSE - suggestion transmission for performing certain tasks. In response to the $\boldsymbol{Q U E R} \boldsymbol{Y}$ and $\boldsymbol{R E Q U E S T}$ messages, the agent receiver sends a response with a message $\boldsymbol{R E P L Y}$, while PROPOSE to received message answers with message AGREE or REFUSE, (Fig. 5).

The JADE agent environment enables the mobility of agents. Agent mobility is performed in classes that implement the MobilityManager interface. The term mobility of agents within MAP and the mobility of agents outside the parent MAP is described in detail [41].

In the SCPWS concept, mobility as an agent characteristic is defined and assigned to service agents. Such feature enables the connection of ESCPWS to collaborative environments and linking to higher management levels in CPPS.

An illustrative example of establishing MAP and agent structures is presented in the next section.

\subsection{1 llustrative Example of ESCPWS in the Laboratory Environment}

In this Section an example of establishing a multiagent platform for connecting the socio, cyber, and physical environment into a functional product unit is presented. For the example implementation, information and communication infrastructure the Experimental Autonomous Cell (EAC) was used, that was developed in LAKOS Laboratories ${ }^{1}$ to support global manufacturing networks. Infrastructure was developed with the JADE software library, and provides a graphical interface for easy creation and operation of multi-agent platforms and agent structures. The basic physical element EAC is the Lakos 150 machine, which was developed at the University of Ljubljana, Faculty of Mechanical Engineering. Engraving Lakos 150 is a machine that allows milling, engraving and drilling of soft materials. Machine Controller Lakos 150 is a PC with Ubuntu operating system and EMC2 online software package.

Fig. 7 shows the graphical user interface LAKOS EAC with the established multi-agent platform where the agents exist.

\footnotetext{
${ }^{1}$ LAKOS - Laboratory for Control and Manufacturing Systems,

University of Ljubljana, Faculty of Mechanical Engineering
} 
The platform is set up on a PC with a public IP address, allowing you to connect to the platform from anywhere. On this basis, the entire EAC system is distributed. Graphic user interface the LAKOS EAC is the main program base that allows you to connect to a platform and create individual agents. Each agent has its own user interface, which is implemented as a pane.

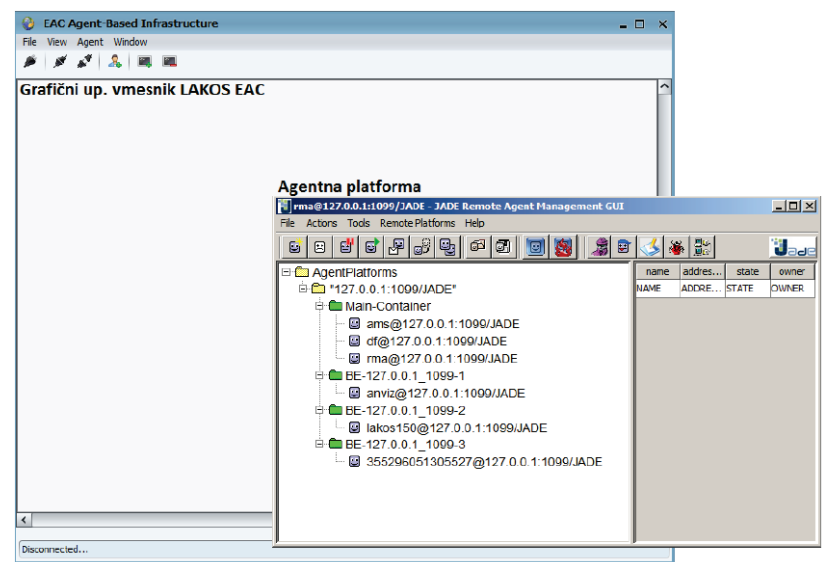

Figure 7 Graphic user interface LAKOS EAC with multi-agent platform [43]

To connect the engraving machine Lakos 150 to the multi-agent based cyber system, a software agent L150Agent was developed. Program code of L150Agent program agent is developed within the developmental program environment of Netbeans (Fig. 8).

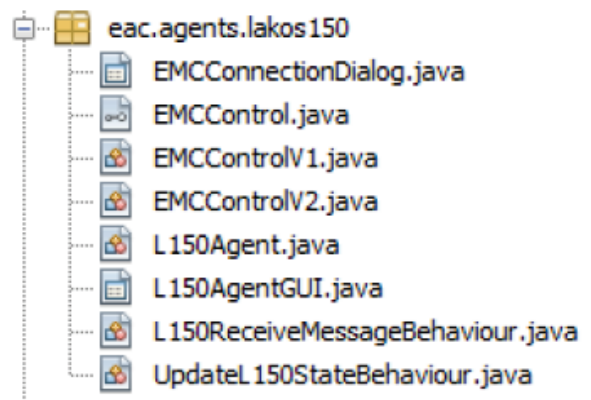

Figure 8 Program code of the L150Agent program agent

For the communication between the L150Agent program agent and controller of engraving machine Lakos 150,EMCControl.java, EMCControlV1 and EMCControlV2, take care, in which functions are gathered for connection and transmission of directions for Lakos 150 control. Class L150Agent.java defines the action of the L150Agent program agent. The graphical user interface of the L150Agent program agent is defined in the class L150AgentGUI.java.

The EMCConnectionDialog.java class allows you to create a new L150Agent agent in a multi-agent platform.

The agent behavior upon receiving the message is written in the class L150ReceiveMessageBehaviour.java. For the presented example it is defined that the agent upon the receipt of queries $\boldsymbol{Q} \boldsymbol{U} \boldsymbol{E} \boldsymbol{R} \boldsymbol{Y}$ corresponds with a record of information about the current state of the LAKOS 150 engraving machine. Through the class L150StateBehavour.java, a cyclical agent management is set up, enabling programmer L150Agent to download data from the Lakos 150 engraving machine at defined time intervals
Connecting a Subject with a cyber system, according to the principles of the SCPWS concept, was accomplished through the development of an appropriate user interface.

The user interface was created as a mobile application for devices running the Android operating system. The SmartLab mobile application structure is created within the Eclipse programming environment. Through communication between program agent L150Agent and mobile apps named SmartLab, the Subject receives information about the status of the engraving machine Lakos 150, located in the physical environment.

In Fig. 9, the graphical user interfaces of mobile application $\mathbf{S m a r t} \boldsymbol{L} \boldsymbol{a b}$ are displayed.

The illustrated example of establishing a multi-agent platform and agent structure, such as the L150Agent program agent and the SmartLab mobile application agent, describes the ability to implement the developed cyber system of CPPS. In the example provided it is possible for the Subject to monitor the state of the process, which is implemented in real time, during the production process itself on the Lakos 150 engraving machine.

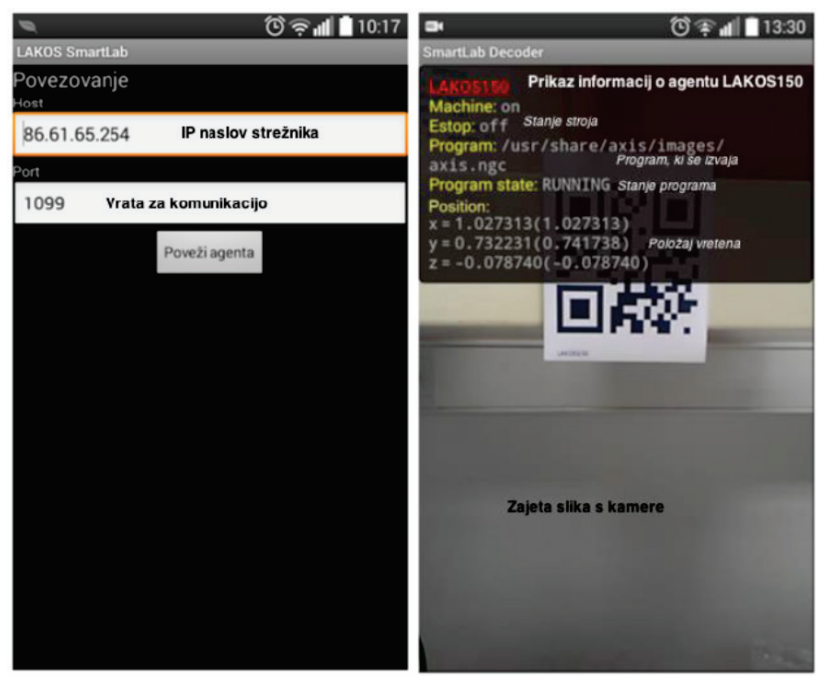

Figure 9 Graphical User Interfaces of apps SmartLab [43]

Through the described example, the proposed concept of the ESCPWS was confirmed and enabled to be transmitted to real industrial environments.

\section{$5 \quad$ ESCPWS in Die Casting Cell}

To illustrate the possibility of introducing the concept ESCPWS in the field of contemporary industrial production Section 5 shows the production area of high pressure die casting (HPDC).

High pressure die casting is an important process in the manufacturing of high volume and low cost metal components for the automotive, household products and electronics industries [36]. It represents a highlyproductive manufacturing process for whose execution very complicated and expensive tools are used. High precision and quality are two key, target factors in the HPDC. To achieve these objectives timely and adequate management of the production process, as well as advanced management of the entire manufacturing system for HPDC are necessary. 
A lot of authors pay attention to the quality control in the HPDC process [44-46].

For the analysis of the current situation in a cell for die casting it is pointed in this section to the indicative data from CoCAST data base. The project CoCAST [47] is developed and integrated module for collecting production data and web control production cell for high-pressure casting molds.

For the analysis of the cell for die casting significant data are recorded in the form of an alarm (different delays of the production process). Alarms are described from a different point of view, such as a longer hour period, their frequency and intensity of the stochastic. For the analysis of alarms data are used on alarm conditions which are collected in the period from 07 January to 30 June for a die casting cell. In the period of 6 months, it was observed that in CoCAST data base 6121 alarms is connected with parameters of the process performed by the die casting machine: time of phase1 (930 alarm), velocity of phase2 (1050 alarms), pressure at phase3 (139 alarm), pressure at closing (816 alarm), thickness of tablets (3174 alarms).

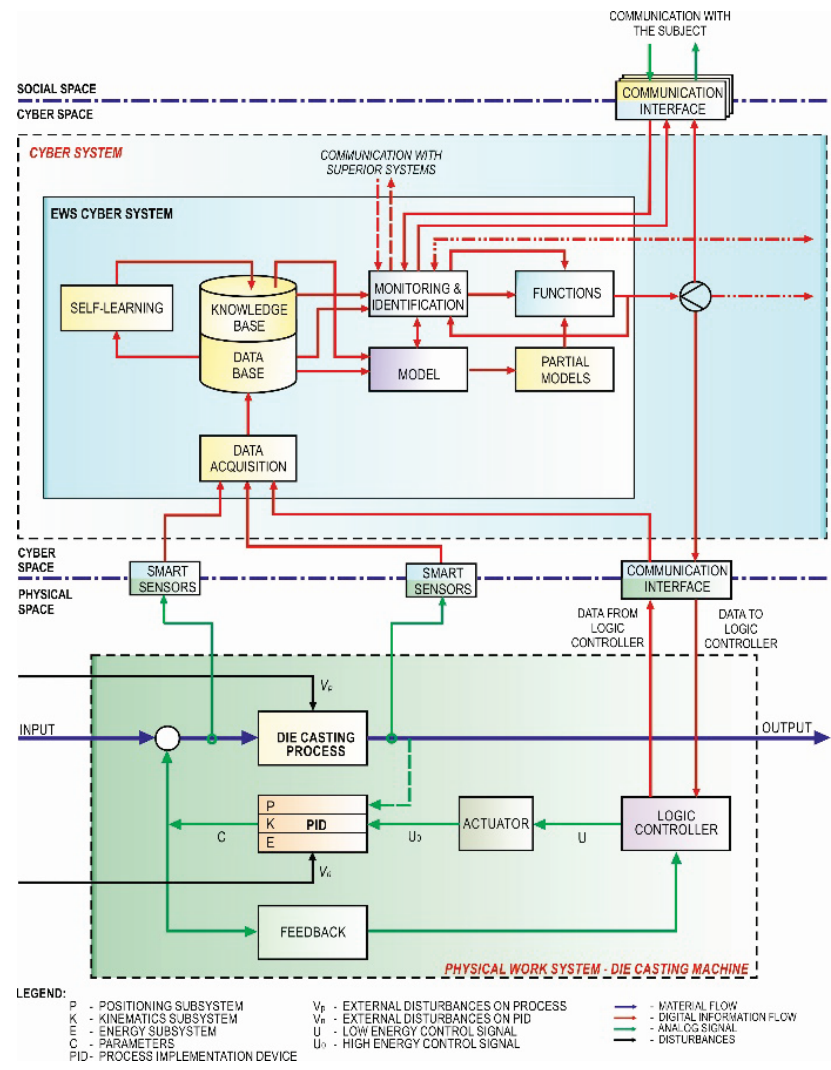

Figure 10 The model of ESCPWS for a die casting

Detailed analysis of data for the alarms described in CoCAST data base comes to the conclusion that it is necessary to improve the process of production castings bringing the correlation values of individual parameters within a die casting machine, or the elementary work system for a die casting.

This implies that it is necessary to enable timely adaptation of process parameters during the execution of the production process. In traditional systems adaptation parameters do the Subject direct to controll the individual machine, after the alarm occurs and stops the process of production. This activity of the Subject requires markedly during the system is in standby no adequate work until the establishment of the appropriate parameters of the production process. The concept SCPWS introduces an advanced method of adaptation of process parameters.

In Fig. 10, the possibility of introducing the concept of ESCPWS in the field of HPDC is presented.

The model of ESCPWS for HPDCis structured based on the generic model of ESCPWS (Section 3) with the three constitutive elements: the Subject, the physical work system of HPDC, and cyber system of the ESCPWS for HPDC. For the presentation of the concept of ESCPWS, the high pressure casting machine is shown in Fig. 10.

The ESCPWS concept enables the adaptation process parameters after each cycle and in the real time. The concept of adapted process parameters is presented in Section 3.

Fig. 11 presents IDEF0 diagram for the adaptive process control in the ESCPWS for die casting. In the cyber system of ESCPWS for die casting three the key functions exist: the monitoring function, prediction function and selfadaptation function. The performance of these functions allows the adaptation of the process parameters in the cyber system of ESCPWS for a die casting.

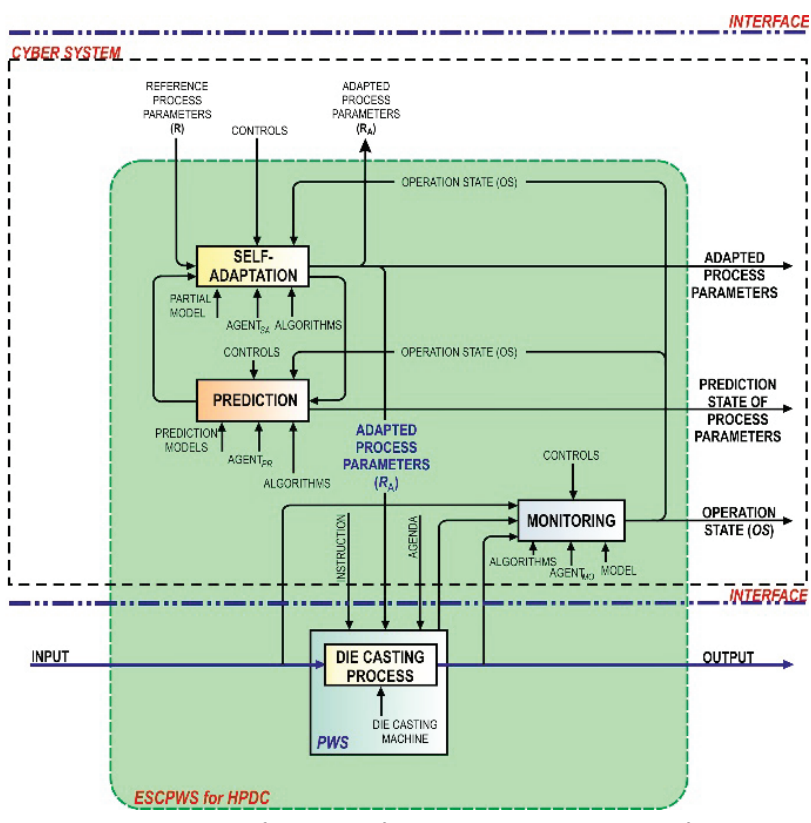

Figure 11 IDEF0 diagram of the adaptive process control function

The mechanisms for implementing functions in the cyber system ESCPWS for a die casting are the agent structures. The dynamic interaction of the agents in realtime enables the adaptation of process parameters in the real time.

For illustration of these concepts of ESCPWS and the adaptive function in the field of HPDC, a portion of data was extracted from the CoCAST database. In the CoCAST database, knowledge models are generated through the self-learning function. The knowledge models are formed on the basis of rules and target values [36].

In order to calculate the new reference values for input process parameters in the next process cycle, the selfadaptation agent $\left(\right.$ Agent $\left._{\mathrm{SA}}\right)$ must know the values of the process parameters of the last process cycle. $\left(\right.$ Agent $\left._{\mathrm{SA}}\right)$ gets these values from the monitoring agents $\left(\right.$ Agent $\left._{\mathrm{MO}}\right)$.

The actual values of process parameters indicate the selection of the corresponding rules that will be used to 
calculate the new reference values for the input parameters of the HPDC process (time of phase1, velocity of phase 2, and presure at phase 3 ).

In this section, for example, let us assume that the measured values of the last process cycle are presented in Tab. 1.

Table 1 The measured values of the last process cycle [36]

\begin{tabular}{|l|c|}
\hline \multicolumn{1}{|c|}{ Attribute } & Value \\
\hline TimeOfPhase1 & $1,905 \mathrm{~ms}$ \\
\hline PressureAtReturn & $90,10 \mathrm{bar}$ \\
\hline VelocityOfPhase2 & $2,88 \mathrm{~m} / \mathrm{s}$ \\
\hline PressureAtPhase3 & $355,04 \mathrm{bar}$ \\
\hline PressureAtClosing & $96,67 \mathrm{bar}$ \\
\hline TemperatureOfOil & $37,93{ }^{\circ} \mathrm{C}$ \\
\hline ThicknessOfTablet & $20,60 \mathrm{~mm}$ \\
\hline
\end{tabular}

Based on the knowledge models [36], the selfadaptation agent calculates the TimeOfPhase1. The formula for calculation of the TimeOfPhase 1 is:

TimeOfPhase $1=0,5689 \times$ Pr essureAt Return

$+1036,8366 \times$ VelocityOfPhase $2+67,4787$

$\times$ Pr esureAtPhase $3-0,0899 \times$ Pr essureAtClo sing

$-15,4466 \times$ TemperatureOfOil $+20,4164$

$\times$ ThicknessOfTable-24902,1816

Analogously, the formula for calculation of the VelocityOfPhase2 is:

VelocityOfPhase $2=0 \times$ Pr essureAt Return

$-0 \times$ TimeAtPhase $1-0,0001 \times$ Pr essureAtPhase 3

$-0,002 \times$ Pr essureAtClo sin $g+0,0003$

$\times$ TemperatureOfOil-0,004×ThicknessOfTablet

$+2,9162$

The last calculation is performed for PressureAtPhase3. Based on the knowledge model, the agent self-adaptation calculates the PressureAtPhase3. For the calculation self-adaptation agent uses the formula for calculation of the PressureAtPhase 3 :

\section{Pr essureAtPhase $3=0 \times$ Pr essureAt Return \\ $-0 \times$ TimeAtPhase $1-0,0029 \times$ VelocityOfPhase 2 \\ $-0,0002 \times$ ThicknessOfTablet $+355,02$}

The new values of the adapted input process parameters are:

$$
R_{\mathrm{A}}=\left\{\begin{array}{c}
\text { TimeOfPhase } 1=1866,812 \mathrm{~ms} \\
\text { VelocityOfPhase } 2=2,97 \mathrm{~m} / \mathrm{s} \\
\text { PressureAtPhase } 3=355,08 \mathrm{bar}
\end{array}\right\}
$$

The agent self-adaptation sends the new values of the adapted input process parameters $\left(R_{\mathrm{A}}\right)$ to the device agent. The device agent delivers the new reference values to the logic controller of the physical work system - die casting machine for the next process cycle.
The presented case clearly indicates the possibility of using the developed SCPWS concepts in the industrial environment.

\section{CONCLUSIONS}

Advanced manufacturing technology opens new possibilities and potentials in design, development, management and control of manufacturing systems. Today, the manufacturing industry is facing the technologies of new industrial revolution - Industry 4.0 and new models CPPS.

This paper presents the development of the concept of the socio-cyber-physical work systems (SCPWS) for Industry 4.0.

First, in the paper the new model is structured from ESCPWS based on restructuring the EWS in the spirit of CPS. Then, the cyber system of EWS is introduced and defined.

The EWS cyber system has enabled the development of new functions for management and control of ESCPWS. The main advantage of the new model is that the elements of the cyber system of EWS enable the management and control of manufacturing systems in the real-time, through the realization of the digitalized and cybernated functions of ESCPWS. The cybernated function of self-adaptation in the ESCPWS is presented in this paper.

Multi-agent technology is used for the implementation of the developed SCPWS and ESCPWS.

The communication between physical and cyber environments enables the management and control of ESCPWS. Such style of management and control in the SCPWS concept allows the development of the function of self-adaptive process control based on the integration of socio, cyber, and physical environments into a single functional entity. The potencial implementation of the developed concept is presented in Section5.

The implementation of the concepts SCPWS in real industrial environment is a challenge for further research in the domain of the cyber-physical production systems.

\section{Acknowledgments}

This work was supported by the Public Scholarship, Development, Disability and Maintenance Fund of the Republic of Slovenia No. 11011-79/2013.

\section{REFERENCES}

[1] Kegermann, H., Wahlster, W. \& Johannes, H. (2013). Recommendations for implementing the strategic initiative INDUSTRIE 4.0. Final report of the Industrie 4.0 Working Group. Forschungsunion, acatech, Technical Report. 78.

[2] Brettel, M., Friederichsen, N., Keller, M. \& Rosenberg, N. (2014). How virtualization, decentralization and network building change the manufacturing landscape: An Industry 4.0 Perspective. World Academy of Science, Engineering and Technology: International Journal of Information and Communication Engineering, 8(1), 37-44.

[3] Gajsek, B., Marolt, J., Rupnik, B., Lerher, T., \& Sternad, M. (2019). Using Maturity Model and Discrete-Event Simulation for Industry 4.0 Implementation. International Journal of Simulation Modelling, 18(3), 488-499. https://doi.org/10.2507/IJSIMM18(3)489 
[4] Bauernhansl, T. (2013). Industry 4.0: Challenges and Opportunities for the Automation Industry. $7^{\text {th }} E F A C$ Assembly Technology Conference.The European Business Conference for Assembly and Handling Technology, January 18/19, 2013, Davos, Switzerland.

[5] Bauernhansl, T., ten Hompel, M. \& Vogel-Heuser, B. (2014). Industrie 4.0 in Produktion, Automatisierung und Logistik. Springer Fachmedien Wiesbaden. https://doi.org/10.1007/978-3-658-04682-8

[6] Vieira, A. A. C., Dias, L. M. S., Santos, M. Y., Pereira, G. A. B., \& Oliveira J. A.(2018). Setting an Industry 4.0 Research and Development Agenda for Simulation-a Literature Review. International Journal of Simulation Modelling, 17(3), 377-390. https://doi.org/10.2507/IJSIMM17(3)429

[7] Jovane, F., Koren, Y., \& Boer, C. R. (2003). Present and Future of Flexible Automation: Towards New Paradigms. CIRP Annals - Manufacturing Technology, 52(1), 543-560. https://doi.org/10.1016/S0007-8506(07)60203-0

[8] Monostori, L. (2014). Cyber-physical Production Systems: Roots, Expectations and R\&D Challenges. Procedia CIRP, (17), 9-13. https://doi.org/10.1016/j.procir.2014.03.115

[9] Gill, H. (2006). NSF perspective and status on cyberphysical systems. In National Workshop on Cyber-physical Systems, October 16-17, 2006. Austin, TX. National Science Foundation.

[10] Ribeiro, L. (2017). Cyber-physical production systems design challenges. 2017 IEEE $26^{\text {th }}$ International Symposium on Industrial Electronics (ISIE), Edinburgh, UK, 1189-1194. https://doi.org/10.1109/ISIE.2017.8001414

[11] Butala, P. \& Sluga, A. (2002). Dynamic structuring of distributed manufacturing systems. Adv. Eng. Informatics, 16(2), 127-133. https://doi.org/10.1016/S1474-0346(02)00007-1

[12] Hozdić, E., Kozjek, D., \& Butala, P. (2020). A cyberphysical approach to the management and control of manufacturing systems. Strojniški Vestnik. - Journal of Mechanical Engineering, 66(1), 61-70. https://doi.org/10.5545/sv-jme.2019.6156

[13] Butala, P. \& Sluga, A. (2006). Autonomous work systems in manufacturing networks. CIRP Annals - Manufacturing Technology, 55(1), 521-524. https://doi.org/10.1016/S0007-8506(07)60473-9

[14] Peklenik, J. (1995). Complexity in Manufacturing Systems. CIRP Journal of Manufacturing Systems, (24), 17-25.

[15] Miorandi, D., Sicari, S., De Pellegrini, F., \& Chlamtac, I. (2012). Internet of things: Vision, applications and research challenges. Ad Hoc Networks, 10(7), 1497-1516. https://doi.org/10.1016/j.adhoc.2012.02.016

[16] Sundmaeker, H., Guillemin, P., Friess, P., \& Woelffle, S. (2010). Vision and challenges for realising the Internet of Things. Publications Office of the European Union, 2010.

[17] Xu, X. (2012). From cloud computing to cloud manufacturing. Robotics and Computer-Integrated Manufacturing, 28(1), 75-86. https://doi.org/10.1016/j.rcim.2011.07.002

[18] Mell, P. \& Grance, T. (2009). The NIST Definition of Cloud Computing. National Institute of Standards and Technology NIST. Gaithersburg, MD, 20899-8930.

[19] Benedikt, M. (1991). Cyberspace: First Steps. The Mit Press. First Printing edition (November, 1991).

[20] The Department of Defense, National Security Presidential Directive 54/Homeland Security Presidential Directive 23 (NSPD-54/HSPD-23), (2008). The Definition of Cyberspace. The White House, Washington, January 8, 2008

[21] Lorents, P., Ottis, R., \& Rikk, R. (2009). Cyber Society and Cooperative Cyber Defence. International Conference on Internationalization, Design and Global Development IDGD 2009: Internationalization, Design and Global
Development, LNCS 5623, 180-186, Springer-Verlag Berlin Heidelberg 2009. https://doi.org/10.1007/978-3-642-02767-3_20

[22] Shen, W., Hao, Q., Yoon H. J., \& Norrie, D. H. (2006). Applications of agent-based systems in intelligent manufacturing: An updated review. Advanced Engineering Informatics, 20(4), 415-431 https://doi.org/10.1016/..aei.2006.05.004

[23] Sluga, A., Butala, P., \& Bervar, G. (1998). A multi-agent approach to process planning and fabrication in distributed manufacturing. Computers \& Industrial Engineering, 35(34), 455-458. https://doi.org/10.1016/S0360-8352(98)00132-6

[24] Leitão, P. (2009). Agent-based distributed manufacturing control: A state-of-the-art survey. Engineering Applications of Artificial Intelligence, 22(7), 979-991. https://doi.org/10.1016/j.engappai.2008.09.005

[25] Leitão, P., Colombo, A. W., \& Karnouskos, S. (2016). Industrial automation based on cyber-physical systems technologies: Prototype implementations and challenges. Computer in Industry, 81, 11-25. https://doi.org/10.1016/j.compind.2015.08.004

[26] Leitao, P., Karnouskos, S., Ribeiro, L., Lee, J., Strasser, T., \& Colombo, A. W. (2016). Smart Agents in Industrial Cyber-Physical Systems. Procedings of the IEEE, 104(5), 1086-1101. https://doi.org/10.1109/JPROC.2016.2521931

[27] Lee, E. A. (2008). Cyber Physical Systems: Design Challenges. International Symposium on Object/ Component/Service-Oriented Real-Time Distributed Computing (ISORC), 2008, 363-369. https://doi.org/10.1109/ISORC.2008.25

[28] Vogel-Heuser, B., Bayrak, G., \& Frank, U. (2011). Agenda CPS - scenario Smart Factory.Increased Availability and Transparent Production, Kassel, 2011.

[29] Monostori L., Kádár, B., Bauernhansl, T., Kondoh, S., Kumara, S., Reinhart, G., Sauer, O., Schuh, G., Sihn, W., \& Ueda, K., (2016). Cyber-physical systems in manufacturing. CIRP Annals-Manufacturing Technology. 65(2), 621-641. https://doi.org/10.1016/j.cirp.2016.06.005

[30] Váncza, J., Monostori, L., Lutters, D., Kumara, S. R., Tseng, M., Valckenaers, P., \& Van Brussel, H. (2011). Cooperative and responsive manufacturing enterprises. CIRP Annals Manuf. Technol, 60(2), 797-820. https://doi.org/10.1016/j.cirp.2011.05.009

[31] Kühnle, H. \& Bitsch, G. (2015). Elements of Manufacturing Networks, Cyber-Physical Production Systems and Smart Automation. Foundations \& Principles of Distributed Manufacturing. Springer International Publishing, 2015. https://doi.org/10.1007/978-3-319-18078-6 5

[32] Liu, Q., Chen, J., Liao, Y., Mueller, E., Jentsch, D., Boerner, F., \& She, M. (2015). An Application of Horizontal and Vertical Integration in Cyber-Physical Production Systems. International Conference on Cyber-Enabled Distributed Computing and Knowledge Discovery, 2015, 110-113. https://doi.org/10.1109/CyberC.2015.22

[33] Stock, T. \& Seliger, G. (2016). Opportunities of Sustainable Manufacturing in Industry 4.0. Procedia CIRP, 40, 536-541. https://doi.org/10.1016/j.procir.2016.01.129

[34] Schuhmacher, J. \& Hummel, V. (2016). Decentralized Control of Logistic Processes in Cyber-physical Production Systems at the Example of ESB Logistics Learning Factory. Procedia CIRP, 54, 19-24. https://doi.org/10.1016/j.procir.2016.04.095

[35] Hozdić, E. (2020). Socio-Cyber-Physical Systems Alternative for Traditional Manufacturing Structures. In: Karabegović I. (eds) New Technologies, Development and Application II. NT 2019. Lecture Notes in Networks and Systems, vol 76. Springer, Cham. https://doi.org/10.1007/978-3-030-18072-0_2

[36] Žapčević, S. \& Butala, P. (2013). Adaptive process control based on a self-learning mechanism in autonomous 
manufacturing systems. The International Journal of Advanced Manufacturing Technology, 66(9-12), 1725-1743. https://doi.org/10.1007/s00170-012-4453-0

[37] Monostori, L., Vancza, J., \& Kumara, S. R. T. (2006). Agent - based systems for manufacturing. CIRP AnnalsManufacturing Technology, 55(2), 697-720. https://doi.org/10.1016/j.cirp.2006.10.004

[38] Kruger, K. \& Basson, A. (2013). Multi-agent Systems vs IEC 61499 for Holonic Resource Control in Reconfigurable Systems. Procedia CIRP, 7, 503-508. https://doi.org/10.1016/j.procir.2013.06.023

[39] Christensen, J. H. (2003). HMS/FB architecture and its implementation. In: Deen, S. M. (eds) Agent Based Manufacturing. Advanced Information processing. Springer Berlin, Heidelberg, 53-87. https://doi.org/10.1007/978-3-662-05624-0_4

[40] Vrabič, R., Škulj, G., Sluga, A., \& Butala, P. (2011). Towards Ubiquitous Manufacturing Systems: ICT Infrastructure for a Global Manufacturing Network. $44^{\text {th }}$ CIRP Conference on Manufacturing Systems, 2011, 1-6.

[41] FIPA, (2014). The Foundation for Intelligent Physical Agents. http://www.fipa.org/

[42] Bellifemine, F. L., Caire, G., \& Greenwood, D. (2007). Developing multi - agent systems with JADE. John Wiley and Sons. https://doi.org/10.1002/9780470058411

[43] Goropevšek, M. (2014). Povezovanje kibernetsko-fizikalnih sistemov $v$ pametnem laboratoriju. (Slovenian language) Diplomska naloga. Fakulteta za strojništvo Ljubljana, 2014.

[44] Syrcos, G. P. (2003). Die casting process optimization using Taguchi methods. Journal of Materials Processing Technology, 135(1), 68-74. https://doi.org/10.1016/S0924-0136(02)01036-1

[45] Rai, J. K., Lajimi, A. M., \& Xirouchakis, P. (2008). An intelligent system for predicting HPDC process variables in interactive environment. Journal of Materials Processing Technology, 203(1-3), 72-79. https://doi.org/10.1016/j.jmatprotec.2007.10.011

[46] Yarlagadda, P. K. D. V. \& Chiang, E. C. W. (1999). A neural network system for the prediction of process parameters in pressure die casting. Journal of Materials Processing Technology, (89-90), 583-590. https://doi.org/10.1016/S0924-0136(99)00071-0

[47] Sluga, A., Butala, P., \& Peklenik, J. (2005). A Conceptual Framework for Collaborative Design and Operations of Manufacturing Work Systems. CIRP Annals-Manufacturing Technology, 54(1), 437-440.

https://doi.org/10.1016/S0007-8506(07)60139-5

\section{Contact information:}

Elvis HOZDIĆ, PhD candidate

(Corresponding author)

University of Ljubljana,

Faculty of Mechanical Engineering,

Askerceva 6, SI-1000 Ljubljana, Slovenia

E-mail: ehozdic@yahoo.com

Peter BUTALA, PhD

Tshwane University of Technology,

Department of Industrial Engineering,

Pretoria, South Africa

E-mail: Peter.Butala@fs.uni-lj.si 